\title{
Protecting at risk cadres of health workers from medical transmission of HIV and Hepatitis B and $C$ through injection safety interventions
}

\author{
Susana de la Torre ${ }^{1 *}$, Innocent Gasimbi ${ }^{2}$, Deepa Bhat ${ }^{1}$, Jessica Posner ${ }^{1}$, Megan Noel $^{1}$, Victoria Masembe ${ }^{3}$, \\ Jackson Songa ${ }^{4}$, lqbal Hossain ${ }^{1}$
}

From $16^{\text {th }}$ International Symposium on HIV and Emerging Infectious Diseases

Marseille, France. 24-26 March 2010

\section{Background}

The Making Medical Injection Safer (MMIS) project funded by the President's Emergency Plan for AIDS Relief implemented by the Ministries of Health and John Snow Inc. aimed to improve injection safety and healthcare waste management practices in eleven countries between 2004 and 2009. MMIS engaged in training and capacity building for healthcare workers, including waste handlers, as well as commodity and logistics support, behavior change communications, and advocacy for safe injection and waste management policies. Waste handlers are considered a highly vulnerable and somewhat ignored group in the infection control chain. Injuries from contaminated needles are a primary route through which blood borne pathogens such as HIV, hepatitis $B$ and $C$ are transmitted in healthcare settings. Percent of reported needlestick injuries, immunization against hepatitis $B$, and knowledge of disease transmission are key indicators related to occupational safety for waste handlers.

\section{Methods}

Cross-sectional studies were carried out to evaluate progress in these areas. Through baseline and follow-up surveys at the intervention sites, observations of waste management and interviews were carried out with waste handlers.

\section{Results}

Waste handlers were asked if they are aware of diseases transmitted through needle stick injuries. Surveys across

\footnotetext{
* Correspondence: mkpahl@gmail.com

'John Snow Inc., Arlington, USA

countries showed that majority are aware of HIV, but not Hepatitis B or C. During the 2008 survey in Kenya for example, $91 \%$ of waste handlers mentioned HIV, but only 33\% mentioned Hepatitis B, and 5\% Hepatitis C. Only three waste handlers reported receiving all three doses of the Hepatitis B vaccination in Kenya while in Uganda, only one waste handler interviewed was fully vaccinated at follow-up. The percent of waste handlers who reported having personal protective equipment varied widely between countries, with $55 \%$ in Kenya reporting having heavy duty gloves and boots, while only $7 \%$ of waste handlers reporting the same in Haiti.

\section{Discussion}

As waste handlers are exposed to blood-born pathogens, there is an urgent need to promote hepatitis B immunization programs which will ensure proper inoculation. Protecting waste handlers through training on proper waste management techniques and provision of personal protective equipment to handle medical waste helps reduce needle stick injuries. All of these are vital for preventing transmission of blood-born pathogens.

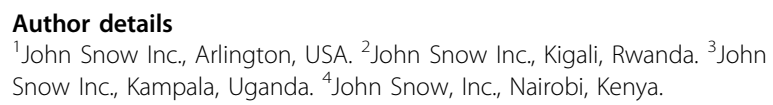

Published: 11 May 2010

doi:10.1186/1742-4690-7-S1-P152

Cite this article as: de la Torre et al:: Protecting at risk cadres of health workers from medical transmission of HIV and Hepatitis B and C through injection safety interventions. Retrovirology 2010 7(Suppl 1):P152. 